\title{
Interactive comment on "Identify the core bacterial microbiome of hydrocarbon degradation and a shift of dominant methanogenesis pathways in oil and aqueous phases of petroleum reservoirs with different temperatures from China" by Zhichao Zhou et al.
}

Anonymous Referee \#2

Received and published: 25 March 2019

In this manuscript, Zhichao Zhou et al describe the geochemistry and microbial community diversity of several petroleum reservoirs characterized by a broad range of temperatures. For microbial community analyses, the aqueous and oil phases were separated and treated independently. Analyses were essentially based on 16S rRNA and mcrA gene sequencing. In addition, qPCR for the mcrA gene were done, although the results of this analysis do not appear to be extensively discussed in the main text. A major conclusion of the work is that despite the distinct geographical locations and

Printer-friendly version

Discussion paper 
different physical-chemical parameters, the analyzed reservoirs shared a common microbiome represented by a small number of OTUs of high relative abundance. Shifts of the methanogenesis mechanisms between the aqueous and oil phase were observed in low and high temperature reservoirs (but not in those of moderate temperature). Overall, the findings are interesting, but there are, however, several aspects that need more careful consideration. The Introductions makes the case for MEOR: however, in the study rationale (line 95-98) there is not apparent connection to MEOR, and this is also not mentioned again in the conclusions. Is there an impact of the findings of this work on MEOR? Could the observed similarities between the microbial communities of the reservoirs be actually caused by the many years of enhanced oil recovery applied at those sites? Overall, the manuscript needs some revision with respect to writing. For some sentences, it is not right away clear if they refer to previous papers, or to results of the present study (for ex. line 30-32, 271-272). It was demonstrated in many other studies that the PCR reaction is introducing biases: clone libraries and MiSeq data should be therefore interpreted as 'relative abundance'. With the exception of methanogenesis, which benefits from mcrA analyses, all other function assignments are done based on short 16S rRNA gene sequences which give taxonomic resolution at phylum level. This should be revised, and sequence data should be more cautiously interpreted. For example, there is no solid basis to assign all those detected phyla as hydrocarbon degraders (line 28-30). It is stated that methanogenesis is a dominant process (Abstract), but there are not too many arguments provided for this; MiSeq analysis show a high relative abundance of Proteobacteria in all samples (Fig. 1), and these are not methanogens. Also, some samples contain very high amounts of sulfate, comparable to sea water (P1 and P5); in the same samples, nitrate is not detectable. If one assumes oxygen is also absent, conditions are favorable for sulfate reduction rather than for methanogenesis. This is not discussed in the manuscript. Currently the reader cannot appreciate if the method used to separate aqueous and oil phases (line 109-110) may alter the community structure. More details should be given, especially the times employed. Was this method tested to make sure cells are not lysed during

Printer-friendly version

Discussion paper
Interactive comment 
heating? For how long was the mixture kept at 50C? What exactly are the 'undetermined results' (line 210-211) that were omitted? 'Core microbiome' is used often in the text: this should be defined. Does it refer to all taxa with a relative abundance over $0.1 \%$ ? What does 'quantity requirement for quality control' means exactly? Other inconsistencies: in the Abstract, enrichment cultures are mentioned (line 29), but there is nothing mentioned about enrichments in the rest of the manuscript; Salmonella and tuberculosis are associated with archaeal communities (line 355 - 359)? The equilibrate claim at line 304-305 is at odds with the conclusion at line 371-372. Figure 3, header is missing for the last column?

Interactive comment on Biogeosciences Discuss., https://doi.org/10.5194/bg-2018-470, 2019. 